\title{
The adaptive significance of unproductive alternative splicing in primates
}

\author{
ADONIS SKANDALIS, ${ }^{1}$ MARK FRAMPTON, ${ }^{1}$ JON SEGER, ${ }^{2}$ and MIRIAM H. RICHARDS ${ }^{1}$ \\ ${ }^{1}$ Department of Biological Sciences, Brock University, St. Catharines, Ontario L2S 3A1, Canada \\ ${ }^{2}$ Department of Biology, University of Utah, Salt Lake City, Utah 84112, USA
}

\begin{abstract}
Alternative gene splicing is pervasive in metazoa, particularly in humans, where the majority of genes generate splice variant transcripts. Characterizing the biological significance of alternative transcripts is methodologically difficult since it is impractical to assess thousands of splice variants as to whether they actually encode proteins, whether these proteins are functional, or whether transcripts have a function independent of protein synthesis. Consequently, to elucidate the functional significance of splice variants and to investigate mechanisms underlying the fidelity of mRNA splicing, we used an indirect approach based on analyzing the evolutionary conservation of splice variants among species. Using DNA polymerase $\beta$ as an indicator locus, we cloned and characterized the types and frequencies of transcripts generated in primary cell lines of five primate species. Overall, we found that in addition to the canonical DNA polymerase $\beta$ transcript, there were 25 alternative transcripts generated, most containing premature terminating codons. We used a statistical method borrowed from community ecology to show that there is significant diversity and little conservation in alternative splicing patterns among species, despite high sequence similarity in the underlying genomic (exonic) sequences. However, the frequency of alternative splicing at this locus correlates well with life history parameters such as the maximal longevity of each species, indicating that the alternative splicing of unproductive splice variants may have adaptive significance, even if the specific RNA transcripts themselves have no function. These results demonstrate the validity of the phylogenetic conservation approach in elucidating the biological significance of alternative splicing.
\end{abstract}

Keywords: alternative splicing; unproductive splice variants; longevity; DNA polymerase $\beta$; primates

\section{INTRODUCTION}

Alternative gene splicing is the process whereby genetic information encoded in DNA sequences is processed into diverse mRNA transcripts, and is a pervasive phenomenon in metazoan, fungal, and plant genomes (Artamonova and Gelfand 2007; Cheah et al. 2007). Alternative splicing (AS) has been best studied in mammals, in which the majority of genes generate multiple alternative mRNA splice variants (SVs) in addition to the canonical transcript (CT), contributing to a vast and complex transcriptome (Yeo et al. 2005; Carninci 2007; Kim et al. 2007a). In humans, up to 95\% of multi-exon genes are alternatively spliced (Nusbaum et al. 2005; Wang et al. 2008), each locus generating an average of 5.4 major transcripts; moreover, large tracts of

Reprint requests to: Adonis Skandalis, Department of Biological Sciences, Brock University, St. Catharines, ON L2S 3A1, Canada; e-mail: adonis.skandalis@brocku.ca; fax: (905) 688-1855.

Article published online ahead of print. Article and publication date are at http://www.rnajournal.org/cgi/doi/10.1261/rna.2127910. non-coding genomic DNA also are transcribed and alternatively spliced (Birney et al. 2007; Tress et al. 2007). Although early reports indicated that the rate of alternative splicing is similar among metazoan organisms (Brett et al. 2002), more recently it has been shown that mammals and birds exhibit similar rates of alternative splicing, but significantly higher rates than in invertebrates or plants (Kim et al. 2004, 2007a). In fact, even among mammals, there is a wide range in rates of alternative splicing, both in terms of the fraction of genes that get alternatively spliced and the number of alternative transcripts per locus (Kim et al. 2004, 2007a).

The adaptive significance of alternative RNA transcript diversity is mostly unknown. Although some variants clearly are "functional" - that is, they are required during the life cycle of the organism and are activated in a regulated manner (Sorek et al. 2004) - the majority do not have obvious functions (Skandalis and Uribe 2004; Sorek et al. 2004). Of 2608 transcripts of protein-coding loci characterized in the Encode project, only 1097 transcripts from 434 loci were predicted to be "productive" and actually 
encode functional proteins (Tress et al. 2007). What, then, is the functional significance of so-called unproductive transcripts that can only code for defective proteins or that cannot code for proteins at all? There is not yet a clear answer to this question, but possible functions of unproductive splice variants (for review, see Blencowe 2006; Artamonova and Gelfand 2007) include regulation of gene expression (Lewis et al. 2003) and gene evolution (Zhang and Chasin 2006; Sorek 2007; Zhang et al. 2007). However, a significant portion of splice variants may simply represent errors (Sorek et al. 2004). In analyses of alternative splicing in humans, random errors apparently do account for splice variants generated by some loci (Skandalis et al. 2002; Skandalis and Uribe 2004).

Two issues complicate the investigation of splice variant (SV) functionality. Firstly, a significant fraction of splice variants is incapable of coding for protein synthesis. As a result, a search for function may need to extend to the possible role of SVs in regulatory pathways, independent of protein synthesis. Secondly, functional analysis of individual splice variants is a very time-consuming process because it is difficult to prove that a transcript does not have any function at any stage during a cell or organism's life. To overcome such complications, a frequently used approach is to analyze genome-wide, evolutionary conservation of alternative splicing (or SV similarity) based on sequences in public databases. Several studies have investigated alternative transcript conservation, as revealed by expressed sequence tag (EST) and microarray surveys, mainly in mammals, but also in birds (chickens), invertebrates, and plants (Kim et al. 2007b). These studies have been valuable in assessing SV conservation, but they have several limitations: they can only use organisms that have been subject to extensive genomics analysis (usually, model organisms); they can be biased by differences in EST or microarray coverage organisms, coverage tending to be considerably lower for non-model organisms; their sensitivity is limited to detecting the major (most frequent) alternative transcripts; and loci that express SVs at lower levels are not well characterized (Skandalis et al. 2002). These limitations may potentially bias our understanding of the mechanisms generating splice variants and their potential function. Techniques based on multiple loci also make the implicit assumption that the functional significance (or lack thereof) of unproductive SVs is the same for all loci, whereas genespecific significance is possible.

We propose an alternative, complementary approach to assessing the extent to which splicing patterns are evolutionarily conserved among different taxa. Whereas the EST and microarray approaches outlined above focus on analyzing limited alternative splicing information from a large number of genes in one or very few taxa, our strategy entails the characterization of alternative splicing in a limited number of indicator loci in multiple, closely related species for which we understand phylogenetic relationships.
DNA polymerase $\beta$ (Pol $\beta$ ) is a ubiquitously expressed housekeeping gene. Pol $\beta$ is a 335 -amino-acid, $39-\mathrm{kDa}$ polymerase encoded by the POLB locus after the splicing of its 14 constitutive exons (Uchiyama et al. 2009; Yamtich and Sweasy 2009). Pol $\beta$ is a member of the X-family of DNA repair polymerases, plays a key role in base excision DNA repair, and its absence leads to sensitivity to methylating agents (Yamtich and Sweasy 2009). POLB is believed to have evolved after the protostome-deuterostome branching, being essential in vertebrates and expressed in all cell types (Uchiyama et al. 2009; Yamtich and Sweasy 2009).

$P O L B$ splicing patterns differ markedly between humans and rats, with humans producing more frequent and more diverse SVs (Disher and Skandalis 2007). These differences suggest that $P O L B$ splicing may have different functions in these two species. Although it is tempting to infer some function by correlating splicing patterns with physiological differences or life history traits, such as body size (large vs. small) or life span (long vs. short), comparisons based on only these two species do not allow us to distinguish whether the differences reflect conserved differences among primates versus rodents or species-specific traits of humans and rats that may not be typical of their groups. Distinguishing among these alternatives requires investigation of the splicing patterns of close relatives.

Therefore, in order to investigate the biological significance of alternative splicing in primates, we characterized SV conservation of the ubiquitously expressed housekeeping gene DNA polymerase $\beta$ ( $\mathrm{Pol} \beta$ ) in five primate species-human (Homo sapiens), chimpanzee (Pan troglodytes), gorilla (Gorilla gorilla), orangutan (Pongo pygmaeus), and macaque (Macaca fascicularis). By comparing phylogenetic patterns of alternative splicing among taxa, we should be able to discern whether splicing patterns are conserved among primates, or whether patterns tend to be species-specific. Because evolutionary conservation implies function, evidence of evolutionary conservation in the frequency or diversity of $P O L B$ splicing would support the hypothesis that production of "unproductive" splice variants has functional significance, even if the function is not yet obvious. Another advantage of the phylogenetic or comparative approach to analyses of the evolutionary conservation of splice variants' diversity and abundance is that it provides a framework for evaluating hypotheses concerning the functional significance of alternative splicing that would not be easily addressed by non-comparative methods. For instance, if splicing patterns are related to physiological or life history traits, such as life span, then such correlations are best evaluated in the context of a known set of phylogenetic relationships (Pagel and Harvey 1989; Freckleton et al. 2002). In this communication, we report that there is little conservation of $P O L B$ SV forms among primates, and that the frequency of alternative splicing of this gene is also significantly different among the five species. On the other hand, splicing frequency and 
diversity correlate well with certain life history parameters, such as longevity. Our results demonstrate that a phylogenetic approach can be valuable in illuminating the functional significance of alternative splicing.

\section{RESULTS}

Using the methodology outlined above, we investigated the conservation of SVs of $P O L B$ among five primate species (Table 1). As POLB coding sequences were not previously known in gorilla and orangutan, we cloned and characterized the POLB coding sequences in these species (GenBank: bankit1263854 GQ900371).

\section{Splice variant characterization}

Characterization of a total of 746 POLB transcripts revealed 25 types of SVs, in addition to the canonical transcript (CT). Of the 25 different SVs found, 24 were unlikely to support functional Pol $\beta$ synthesis, because they were truncated or because the reading frame was altered, leading to missense and/or introduced premature termination codons. One of the 24 non-functional transcripts was $\Delta 2$, which as a result of exon-2 loss, contains a premature stop codon and is incapable of coding for protein (Simonelli et al. 2009). This unproductive transcript was generated in all five species. $\Sigma_{\alpha}$ is the single SV known to support synthesis of a Pol $\beta$ that resembles the CT. It represents an in-frame intron retention event, which has been reported to produce a larger Pol $\beta$ protein, but one without polymerase function (Chyan et al. 1996). It should be noted that the primers used to amplify POLB in primates were complementary to the start and end exons of the canonical primate transcript. The possibility exists that alternative start and end sites exist and that, consequently, additional POLB transcripts may be generated that are not detected in these experiments. However, we believe that this is unlikely since extensive search by us and others has not revealed an alternative start or end site in humans or chimpanzees.

Overall, splice variant frequency and transcript diversity (including CT) were strongly correlated (Pearson $r=0.981$,

TABLE 1. POLB frequency of alternative splicing and transcript diversity among primates

\begin{tabular}{lcccc}
\hline Species & $\begin{array}{c}\text { Total transcripts } \\
\text { surveyed }\end{array}$ & $\begin{array}{c}\text { Number of } \\
\text { splice variants }\end{array}$ & $\begin{array}{c}\text { Splice variant } \\
\left.\text { frequency }^{\mathrm{a}} \%\right)\end{array}$ & $\begin{array}{c}\text { Transcript } \\
\text { diversity }^{\mathrm{b}}\end{array}$ \\
\hline Macaca fascicularis & 169 & 21 & 12.4 & 6 \\
Pongo pygmaeus & 107 & 56 & 52.3 & 12 \\
Gorilla gorilla & 114 & 35 & 30.7 & 9 \\
Pan troglodytes & 119 & 31 & 26.1 & 7 \\
Homo sapiens & 237 & 136 & 57.4 & 14 \\
\hline
\end{tabular}

${ }^{a}$ Splice variant frequency refers to the relative frequency of splice variants compared to the total number of transcripts characterized.

${ }^{\mathrm{b}}$ Transcript diversity refers to the number of different types of transcripts observed. $p=0.0033)$ as expected, but both frequency and diversity of SVs were highly variable among the five primates studied here (Table 1). The proportion of SVs was lowest in Macaca, intermediate in Gorilla and Pan, and highest in Homo and Pongo $\left(\chi^{2}=104.4, \mathrm{df}=4, p<0.0001\right)$. When we compared pairs of lineages from the phylogeny in Figure 1, the two branches below each of the four nodes differed significantly in the proportions of SVs (Node $1, \chi^{2}=31.23$, $p<0.0001$; Node 2, $\chi^{2}=9.26, p<0.01$; Node $3, \chi^{2}=13.04$, $p<0.001$; Node $4, \chi^{2}=25.10, p<0.0001$ ), indicating that there was no particular phylogenetic pattern to the frequency of SVs.

All five species exhibited distinct SV spectra (Table 2). As noted above, only $\Delta 2$ was found in all five, and of the remaining 24 variants, one $(\Delta 12,13)$ was observed in three species, and 23 were found in only one or two species. In other words, the total pool of primate SVs was much more diverse than the subset of SVs exhibited by any particular species. Consequently, the $\mathrm{H} 1$ hypothesis (that the underlying distribution for each species is represented by the pooled splice variant distribution) was rejected for all species except Pongo (Table 3). The $\mathrm{H} 2$ hypothesis (that the underlying distribution for each species is represented by the human distribution) was also rejected, indicating that the diversity of different SVs in Macaca, Gorilla, and Pan was significantly lower than in Homo, whereas Pongo and Homo were similar.

It is unlikely that we sampled the entire possible pool of splice variants. Eight different statistical approaches to estimating the underlying "true" species richness suggest that further sampling could uncover 25-30 additional splice variants among these five primate species (Fig. 2).

\section{Alternative splicing patterns by species}

Homo sapiens

Humans exhibit a distinct $P O L B$ splice variant spectrum characterized by more transcript types and higher overall frequency of SVs. Of 237 POLB transcripts analyzed, 136 $(57.4 \%)$ were SVs of 13 types and $101(43.6 \%)$ were CTs. The predominant SVs were $\Delta 2(25.3 \%), \Delta 11(12.2 \%)$, and $\Sigma \alpha(7.1 \%)$ of all transcripts. Even though SVs with single exon skipping or partial intron retention were the most frequently observed, the majority $(8 / 13)$ of the human SVs types characterized represented complex splicing events involving more than one exon or intron.

\section{Pan troglodytes}

$P O L B$ was found to be alternatively spliced in $26.1 \%$ of 119 chimp POLB transcripts (Table 2). Six variant transcript types were identified, but $\Delta 2$ 


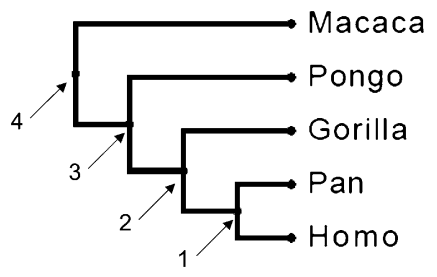

FIGURE 1. Phylogenetic relationships among the five primate species in this study, with numbers indicating statistical comparisons of the proportion of splice variants at bifurcation points. All four comparisons resulted in significant differences, each pair of branches (statistics in text). For instance, node 2 indicates a significant difference in splice variant frequency between Gorilla and Pan + Homo.

predominated (26 of the $31 \mathrm{SVs}$ ). Three SVs demonstrated single-exon skipping or intron retention, and three exhibited complex splicing patterns involving exon 2 .

\section{Gorilla gorilla}

POLB was alternatively spliced in $32.5 \%$ of 117 gorilla transcripts. Gorilla exhibited nine POLB SVs, and it was the only species investigated in which no partial intron retentions were observed. Only one of the transcripts, $\Delta 2$, was the product of single exon skipping, and it was also the most frequent variant observed accounting for $12.8 \%$ of all transcripts. Multiple exon skipping was seen in five of nine transcripts.

\section{Pongo pygmaeus}

POLB was alternatively spliced in $53.6 \%$ of 110 transcripts, similar to that observed in humans. Transcript diversity (11 SVs plus the canonical transcript) was also nearly as high as that seen for human cell lines, but 7/11 SVs were unique to orangutan. $\Delta 2$ was the most frequent SV (19.6\%). Most of the SVs (9/11) represented complex splicing events.

\section{Macaca fascicularis}

Of 169 POLB transcripts, 21 (12.2\%) were SVs of five types. $\Delta 2$ was the most frequent SV $(9.5 \%)$, and macaque was the only species examined in which single exon or intron alternative splicing events outnumbered (3/5) complex events. A complex event, $\Sigma \beta$,pI3, was the first instance of partial intron 3 retention.

\section{Correlation of alternative splicing output with life history parameters}

The relationship between alternative splicing frequency or splice variant diversity and life history parameters is shown in Figure 3. We found that extent of alternative splicing does not correlate well with log female body size (frequency: $r=-0.114$, n.s.; diversity: $r=-0.020$, n.s.), $\log$ standard metabolic rate (frequency: $r=-0.653$, n.s.; diversity: $r=-0.579$, n.s.), correlates weakly with $\log$ gestation length (frequency: $r=0.845, p=0.071$; diversity: $r=0.882$, n.s.), and correlates most strongly with log life span (frequency: $r=0.919, p=0.027$; diversity: $r=0.882$, $p=0.048$ ) and age at first reproduction (frequency: $r=$ 0.902, $p=0.036$; diversity: $r=0.854, p=0.065)$.

\section{DISCUSSION}

$P O L B$ is a highly conserved gene among mammals, with $87 \%$ conservation at the nucleotide level between rats and humans and $99 \%$ among primates, and yet POLB splicing patterns vary considerably within and among species. Our analysis of $P O L B$ alternative splicing revealed significantly different overall frequencies of splice variants among primates, ranging from $12 \%$ to $57 \%$. It also revealed significantly different alternative transcript patterns among primates, reflecting diversity in both the types of transcripts generated and in the relative abundance.

A substantial number of POLB splice variants represented complex splicing events involving skipping or retention of non-contiguous sequences. The complexity of

TABLE 2. Splice variant spectra for DNA polymerase $\beta$ in five primate lineages

\begin{tabular}{|c|c|c|c|c|c|c|}
\hline \multirow[b]{2}{*}{ Transcript } & \multirow[b]{2}{*}{ PPL } & \multicolumn{5}{|c|}{ Number of transcripts observed } \\
\hline & & Масаса & Pongo & Gorilla & Pan & Homo \\
\hline CT & 335 & 148 & 51 & 79 & 88 & 101 \\
\hline$\Sigma \alpha$ & 370 & & & & 1 & 17 \\
\hline$\Sigma \beta$ & 193 & 2 & & & & 3 \\
\hline$\Sigma \alpha, \beta$ & 228 & & & & & 1 \\
\hline$\Sigma \beta, \mathrm{pl} 3$ & 96 & 2 & & & & \\
\hline$\Delta 2, \Sigma \beta$ & 26 & & 2 & & & \\
\hline$\Delta 2$ & 26 & 14 & 21 & 15 & 26 & 60 \\
\hline$\Delta 2,11$ & 26 & & 11 & 8 & & \\
\hline$\Delta 2,3,11$ & 28 & & & & & 2 \\
\hline$\Delta 2,4,5$ & 26 & & & 4 & 1 & \\
\hline$\Delta 2,11-13$ & 26 & & & & & 2 \\
\hline$\Delta 2,13$ & 26 & & 6 & & & \\
\hline$\Delta 2,12,13$ & 26 & & 1 & & 1 & \\
\hline$\Delta 2-6$ & 42 & & 2 & & & \\
\hline$\Delta 2,5$ & 26 & & 4 & & 1 & \\
\hline$\Delta 2-9$ & 44 & & 1 & & & \\
\hline$\Delta 2-5,13$ & 164 & & 1 & & & \\
\hline$\Delta 3-5$ & 268 & 2 & & & & \\
\hline$\Delta 4$ & 310 & & & & & 9 \\
\hline$\Delta 4,5,12,13$ & 71 & & & 1 & & \\
\hline$\Delta 4-10$ & 190 & & & & & 2 \\
\hline$\Delta 4-11$ & 161 & & & & & 1 \\
\hline$\Delta 4-13$ & 93 & & & & & 1 \\
\hline$\Delta 11$ & 306 & & & & 1 & 29 \\
\hline$\Delta 11-13$ & 261 & & & 1 & & 7 \\
\hline$\Delta 12,13$ & 290 & & 2 & 6 & & 2 \\
\hline$\Delta 13$ & 267 & 1 & 5 & & & \\
\hline Total & & 169 & 107 & 114 & 119 & 237 \\
\hline
\end{tabular}

(PPL) Predicted protein length; (CT) canonical transcript. 
TABLE 3. Statistical comparison of observed versus expected rates of $P O L B$ transcript diversity based on the randomization method of Richardson and Richards (2008)

\begin{tabular}{lccccc}
\hline Comparison & Macaca & Pongo & Gorilla & Pan & Homo \\
\hline Observed number of variants & 6 & 12 & 7 & 7 & 14 \\
Expected number of variants & 11.64 & 10.26 & 10.45 & 10.57 & 12.63 \\
Probability under H1 ${ }^{\text {a }}$ & $p<0.01$ & n.s. & $p<0.01$ & $p<0.01$ & $p<0.05$ \\
Probability under $\mathrm{H}^{\mathrm{b}}$ & $p<0.01$ & n.s. & $p<0.01$ & $p<0.01$ & n.s. \\
\hline
\end{tabular}

The expected value is the median of the distribution created by 10,000 iterations of the randomization procedure, given the sample size (observed number of variants) and the null hypothesis.

${ }^{a}(\mathrm{H} 1)$ The underlying distribution of transcript types is the pooled primate distribution.

${ }^{b}(\mathrm{H} 2)$ The underlying distribution of transcript types is the human distribution. analyzing differences in the sequence of predicted exonic splicing regulators between human and chimpanzee, it was reported that sequence changes in these regions are not associated with changes in alternative splicing levels (Irimia et al. 2009). Our results suggest instead that differences in alternative transcript output and transcript diversity may be due either to differential fidelity of the core spliceosomes in the nearly identical splice sites of primates or differences in regulatory splicing factor expression or localization. It is very unlikely that the these variants would not normally be detectable by EST and microarray analyses, and therefore it is possible that transcript diversity may be underestimated in the literature. Nonetheless, our observations agree with findings in a comparative genomics study that used quantitative microarrays of orthologous "cassette" human and chimpanzee exons and reported pronounced splicing-level differences in the two species (Calarco et al. 2007). Importantly, these differences appeared to be independent of the level of transcription. Calarco et al. (2007) did not investigate alternative splicing events that were unique to each genome, citing lack of substantial transcript data for the chimpanzee, but predicted that since there is $99 \%$ sequence identity between the two species, there should be few exons that would be affected uniquely during splicing. In contrast, our data reveal that there is significant POLB transcript diversity among species despite high DNA sequence conservation: of the 27 transcript variants, only two-WT and $\Delta 2$-were observed in all five primates. The evolutionary conservation of $\Delta 2$ is enigmatic, given the overall lack of concordance among species. The $\Delta 2$ transcript does not support protein synthesis (Disher and Skandalis 2007; Simonelli et al. 2009). Possibly it functions by providing a miRNA precursor, although sequence analysis of the truncated transcript and intron1 did not reveal any obvious targets. Interestingly, this transcript is highly up-regulated in some cancers and neurodegenerative disease synthesis (Disher and Skandalis 2007; Simonelli et al. 2009).

Given the rarity of phylogenetically conserved transcripts despite the high degree of $P O L B$ sequence identity among primates, our data support the hypothesis that the generation of diverse splice variants in primates is not the result of differences in control exonic and intronic cis sequences. Indeed, in a study

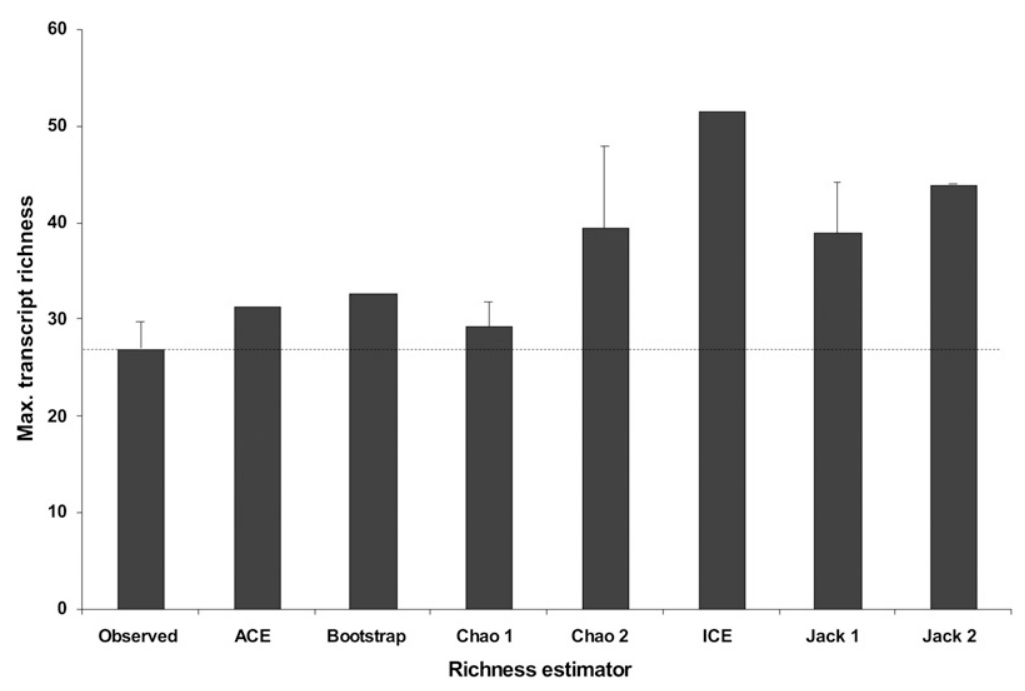

FIGURE 2. Estimated maximum number of $P O L B$ transcripts in the five primate species studied, based on eight different estimation methods implemented in EstimateS diversity software. The dashed line indicates the observed number of transcripts (27). Error bars represent the standard deviation for each predictor based on 100 sampling replicates. 

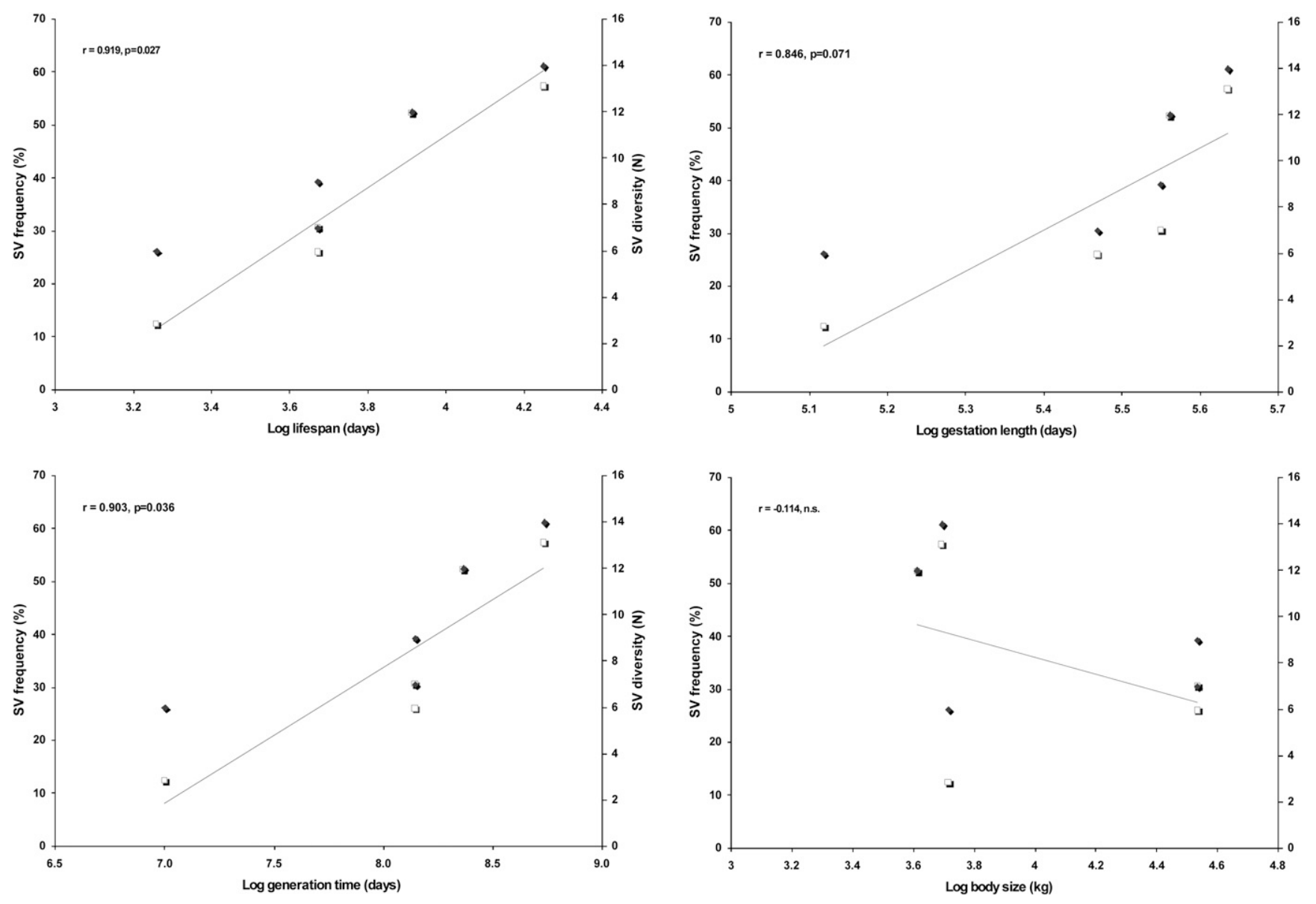

FIGURE 3. Correlation of life history parameters and splicing frequency (white squares) and diversity (gray diamonds). Lines and Pearson $r$ statistics represent correlations based on splicing frequency, but qualitatively similar results are obtained from correlations based on SV diversity, as frequency and diversity are strongly correlated.

generally (data not shown). This observation could indicate that the production of $P O L B$ unproductive splice variants is directly or indirectly related to molecular mechanisms determining species longevity. Although individual POLB splice variants are not evolutionarily conserved in primates, the process that generates them could be related to how long an organism can live. The connection to longevity is especially intriguing given that $P O L B$ is a key enzyme in DNA repair and given that $P O L B$ splicing is regulated by p53, a protein orchestrating cell survival or apoptosis pathways following stress and/or damage (Disher and Skandalis 2007). In human cells, p53 mediates an increase in the frequency of $P O L B$ unproductive splice variants following genotoxic stress (Disher and Skandalis 2007). Unproductive alternative splicing may be a mechanism for down-regulating or attenuating gene expression (Green et al. 2003; Calarco et al. 2007). Interestingly, some types of DNA damage, such as cisplatin-induced lesions, are repaired more slowly in human cells than in chimpanzee cells, which is consistent with the hypothesis of downregulation of repair in humans (Weis et al. 2008). Pol $\beta$ is capable of error-prone bypassing of several types of DNA lesions, including 8-oxo-7,8-dihydrodeoxyguanine, cisplatininduced lesions, and UV-induced lesions (Yamtich and Sweasy 2009). Overexpression of $\mathrm{Pol} \beta$ is associated with an increase in the mutation rate (Chan et al. 2007). The net effect of the unproductive $P O L B$ alternative transcripts would be a reduction in the efficiency of Pol $\beta$ synthesis and consequently a delay in DNA repair and the rate of cell growth. In normal undamaged cells, this would have minimal impact, but in tumors it could retard development and growth significantly, thereby promoting cell senescence and apoptosis.

\section{Conclusion}

Our data on $P O L B$ do not support a direct functional role for specific unproductive alternative transcripts but do support the hypothesis that unproductive alternative splicing in and of itself may be adaptive and regulated. To our knowledge, this is the first demonstration of a correlation between life span and alternative splicing frequency in any 
gene. It is paradoxical that the frequency of apparently unproductive SVs of a locus involved in maintaining DNA integrity would correlate with maximal life span.

These results also demonstrate how a phylogenetic approach-that is, comparing the splicing patterns of one gene among many species, rather than comparing many genes in one or two model species-allowed us to search for conserved features of splice variants that imply adaptive significance, even though the vast majority of splice variants do not produce functional proteins. Future comparative studies may be able to infer adaptive significance from other features of splice variants, such as open reading frames, location in the $3^{\prime}$ or $5^{\prime}$ ends of genes, frequency of synonymous and non-synonymous substitutions among alternatively spliced exons, and length of introns framing alternative spliced exons.

\section{MATERIALS AND METHODS}

\section{Cell lines}

The cell lines used in this project were untransformed fibroblasts that were purchased from Coriell Cell Repositories. The cell lines surveyed were $H$. sapiens (AG05965 and AG08906), P. pygmaeus (AG12256), G. gorilla (AG05251), P. troglodytes (S003644), and M. fascicularis (AG21329). Each cell line was harvested from young animals and had undergone less than eight cell divisions at the time of purchase. Cells were grown in Dulbecco's Modified Eagle's Medium supplemented with $10 \%$ heat-inactivated fetal calf serum as well as antimycotics and antibiotics at $37^{\circ} \mathrm{C}$ and $5 \% \mathrm{CO}_{2}$. Cells were grown to $75 \%-90 \%$ confluence before they were lysed to extract total RNA.

\section{RNA isolation}

Total RNA was extracted using the Sigma GenElute Mammalian Total RNA Kit following the manufacturer's directions. RNA was stored at $-80^{\circ} \mathrm{C}$ until needed.

\section{First-strand synthesis}

First-strand synthesis was carried out using Thermoscript II RTPCR system (Invitrogen) and an oligo(dT) pimer according to the manufacturer's instructions.

\section{DNA polymerase $\beta$ library}

A $P O L B$ transcript library was generated by 15 cycles of polymerase chain reaction amplification of first-strand DNA with primers that were complementary to $P O L B$ sequences of all species investigated (POLBFOR: 5'-CCGCAGGAGACCCTCAACGG; POLBREV: 5' -CCACTGGATGTAATCAAAAATGTC).

\section{Ligation and cloning}

PCR products were ligated into the Promega pGEM T-easy Vector I cloning system. Ligations were incubated for $16-18 \mathrm{~h}$ at $4^{\circ} \mathrm{C}$. Electrocompetent DH5 $\alpha$ cells were transformed with the ligations using a Gene Pulser II (Bio-Rad) electroporator. Cloned transcripts were characterized by sequencing.

\section{Splice variant nomenclature}

SVs with a skipped exon are indicated by the symbol $\Delta$ and the name of the skipped exon or exons. SVs with partial intron retention are indicated by $\Sigma$ and either partial intron (pI) and the intron number or by Greek letters if a letter had been assigned in previous reports (Skandalis and Uribe 2004; Disher and Skandalis 2007).

\section{Statistical analysis of transcript diversity}

We compared proportional frequencies of splice variants among the five primate species using $\chi^{2}$ tests. To compare the number of different types of transcript or transcript diversity among the primates, we adapted methods commonly used in ecological research to compare species diversity among geographic sites, as follows.

In ecology, taxonomic diversity can be measured in terms of proportional abundance, or the relative numbers of individuals of each species type in a community. By the same token, transcript diversity can be assessed in terms of the proportional abundance of each transcript type within a primate species. Observed transcript richness increases non-linearly with sampling effort (more different types of transcripts are found when more are sampled), as illustrated in the following example. We compare two hypothetical primate species, each with a transcript richness of 3 . The "true" transcript distribution in Primate A is $50 \%$ transcript 1, $25 \%$ transcript 2, and 25\% transcript 3, whereas the distribution in Primate B is $95 \%$ transcript 1, 3\% transcript 2, and $2 \%$ transcript 4 . The two primates produce different but overlapping sets of transcripts, and the total richness of both species is 4 . Primate $\mathrm{A}$ is considered to be more diverse because if two transcripts are randomly drawn from its pool, they are more likely to differ, than two transcripts randomly drawn from Primate B's pool. As a result, if only a small proportion of the total transcript population can be sequenced, then the chances of correctly inferring a transcript richness of 3 are higher for Primate A than for B, because some are rarer in Primate B and less likely to be detected.

The above example illustrates why comparisons of transcript diversity among samples must include a correction for sample size, but since the underlying distribution of transcript diversity is unknown for any gene in any species, there is no straightforward correction for sample size. To address this problem, we adopted a randomization approach for comparing observed levels of taxonomic species richness among ecological communities with different sample sizes (Richardson and Richards 2008). The approach employs a re-sampling procedure to generate an expected distribution of transcript richness values for each primate species, given the number of transcripts actually assessed. In this procedure, a set of $\mathbf{N}$ transcripts (where $\mathbf{N}$ is the total number of transcripts assessed in all five primate species of this study) was created representing a null distribution of all 26 transcript types $\left(n_{1}, n_{2}, n_{3} \ldots, n_{26}\right)$, including the canonical transcript (CT). Then each transcript was randomly assigned to 1 of 5 bins, each representing one primate species. The number of transcripts $\left(\mathbf{N}_{1}, \mathbf{N}_{2}, \mathbf{N}_{3}, \mathbf{N}_{4}, \mathbf{N}_{5}\right)$ assigned to each bin was the number of transcripts actually assessed for each primate, so when a bin was 
"filled," no further transcripts could be assigned to that bin. When all $\mathbf{N}$ transcripts had been assigned, the transcript richness of each primate species was assessed by counting the number of different transcript types assigned to each bin. The entire procedure was done 10,000 times, generating an expected distribution of expected transcript richnesses for each primate species, dependent on both the underlying null distribution and the sample size for that species. The observed transcript richness for each primate species was then compared to the expected distribution for that species. The tails of the expected distribution represent transcript richness values with very low probabilities, given the null hypothesis and the sample size for each primate species.

Two null hypotheses were used to generate expected distributions with this procedure. With $\mathrm{H} 1$, we assumed no differences among primate species in the level of transcript diversity. The underlying distribution used for the randomization was thus based on the pooled sample of $\mathbf{N}=746$ transcripts from all five species. With $\mathrm{H} 2$, the underlying distribution used for the randomization was based on that for humans, adjusted proportionally to a total sample size of $\mathbf{N}=746$.

To estimate the number of splice variants not discovered, we again borrowed an approach from ecological sampling, in which a series of estimators can be calculated to suggest how many species remain undiscovered by current sampling. We use the program ESTIMATE-S (Colwell 2006) to calculate the following estimators, treating each primate as an independent sample and each transcript type as a species: Mao Tau (for the standard deviation associated with the observed number of transcripts), ACE (abundance-based coverage estimator), ICE (incidence-based coverage estimator), Chao 1 and 2, Jack-knife first and second order, and bootstrap (Magurran 2004).

To investigate whether more closely related species were more likely to have similar rates of SV production, we compared the proportions of SVs in each pair of branches at each of the four nodes in Figure 1; these contrasts are phylogenetically independent (Felsenstein 1985). We used Pearson correlation coefficients to examine the relationship between splicing frequency (proportion of splice variants) and life history parameters for each primate species taken from Harvey and Clutton-Brock (1985). The parameters used were log female body size, log standard metabolic rate, correlates log gestation length, log life span, and age at first reproduction.

\section{ACKNOWLEDGMENTS}

We thank Ted Gurney for invaluable help with cell culture and Esther Tiedtke for providing transcript sequences. This research was supported by NSERC Discovery grants to A.S. and M.H.R., and a Brock University Undergraduate Research Award to M.F.

Received February 13, 2010; accepted July 12, 2010.

\section{REFERENCES}

Artamonova II, Gelfand MS. 2007. Comparative genomics and evolution of alternative splicing: The pessimists' science. Chem Rev 107: 3407-3430.

Birney E, Stamatoyannopoulos JA, Dutta A, Guigo R, Gingeras TR, Margulies EH, Weng Z, Snyder M, Dermitzakis ET, Thurman RE, et al. 2007. Identification and analysis of functional elements in
$1 \%$ of the human genome by the ENCODE pilot project. Nature 447: 799-816.

Blencowe BJ. 2006. Alternative splicing: New insights from global analyses. Cell 126: 37-47.

Brett D, Pospisil H, Valcarcel J, Reich J, Bork P. 2002. Alternative splicing and genome complexity. Nat Genet 30: 29-30.

Calarco JA, Xing Y, Caceres M, Calarco JP, Xiao X, Pan Q, Lee C, Preuss TM, Blencowe BJ. 2007. Global analysis of alternative splicing differences between humans and chimpanzees. Genes Dev 21: 2963-2975.

Carninci P. 2007. Constructing the landscape of the mammalian transcriptome. J Exp Biol 210: 1497-1506.

Chan K, Houlbrook S, Zhang QM, Harrison M, Hickson ID, Dianov GL. 2007. Overexpression of DNA polymerase $\beta$ results in an increased rate of frameshift mutations during base excision repair. Mutagenesis 22: 183-188.

Cheah MT, Wachter A, Sudarsan N, Breaker RR. 2007. Control of alternative RNA splicing and gene expression by eukaryotic riboswitches. Nature 447: 497-500.

Chyan YJ, Strauss PR, Wood TG, Wilson SH. 1996. Identification of novel mRNA isoforms for human DNA polymerase $\beta$. DNA Cell Biol 15: 653-659.

Colwell RK. 2006. EstimateS-Statistical estimation of species richness and shared species from samples. University of Connecticut. http://viceroy.eeb.uconn.edu/estimates.

Disher K, Skandalis A. 2007. Evidence of the modulation of mRNA splicing fidelity in humans by oxidative stress and p53. Genome 50: $946-953$.

Felsenstein J. 1985. Phylogenies and the comparative method. Am Nat 125: $1-15$.

Freckleton RP, Harvey PH, Pagel M. 2002. Phylogenetic analysis and comparative data: A test and review of evidence. Am Nat 160: 712726.

Green RE, Lewis BP, Hillman RT, Blanchette M, Lareau LF, Garnett AT, Rio DC, Brenner SE. 2003. Widespread predicted nonsensemediated mRNA decay of alternatively-spliced transcripts of human normal and disease genes. Bioinformatics (Suppl 1) 19: i118-i121.

Harvey PH, Clutton-Brock TH. 1985. Life history variation in primates. Evolution 39: 559-581.

Irimia M, Rukov JL, Roy SW. 2009. Evolution of alternative splicing regulation: Changes in predicted exonic splicing regulators are not associated with changes in alternative splicing levels in primates. PLoS ONE 4: e5800. doi: 10.1371/journal.pone.0005800.

Kim H, Klein R, Majewski J, Ott J. 2004. Estimating rates of alternative splicing in mammals and invertebrates. Nat Genet 36: 915-917.

Kim E, Magen A, Ast G. 2007a. Different levels of alternative splicing among eukaryotes. Nucleic Acids Res 35: 125-131.

Kim N, Alekseyenko AV, Roy M, Lee C. 2007b. The ASAP II database: Analysis and comparative genomics of alternative splicing in 15 animal species. Nucleic Acids Res 35: D93-D98.

Lewis BP, Green RE, Brenner SE. 2003. Evidence for the widespread coupling of alternative splicing and nonsense-mediated mRNA decay in humans. Proc Natl Acad Sci 100: 189-192.

Magurran AE. 2004. Measuring biological diversity. Wiley-Blackwell, New York.

Nusbaum C, Zody MC, Borowsky ML, Kamal M, Kodira CD, Taylor TD, Whittaker CA, Chang JL, Cuomo CA, Dewar K, et al. 2005. DNA sequence and analysis of human chromosome 18. Nature 437: 551-555.

Pagel MD, Harvey PH. 1989. Comparative methods for examining adaptation depend on evolutionary models. Folia Primatol (Basel) 53: $203-220$.

Richardson JML, Richards MH. 2008. A randomisation program to compare species-richness values. Insect Conserv Divers 1: 135-141.

Simonelli V, D’Errico M, Palli D, Prasad R, Wilson SH, Dogliotti E. 2009. Characterization of DNA polymerase $\beta$ splicing variants in gastric cancer: The most frequent exon 2-deleted isoform is a noncoding RNA. Mutat Res 670: 79-87. 
Skandalis A, Uribe E. 2004. A survey of splice variants of the human hypoxanthine phosphoribosyl transferase and DNA polymerase $\beta$ genes: Products of alternative or aberrant splicing? Nucleic Acids Res 32: 6557-6564.

Skandalis A, Ninniss PJ, McCormac D, Newton L. 2002. Spontaneous frequency of exon skipping in the human HPRT gene. Mutat Res 501: 37-44.

Sorek R. 2007. The birth of new exons: Mechanisms and evolutionary consequences. RNA 13: 1603-1608.

Sorek R, Shamir R, Ast G. 2004. How prevalent is functional alternative splicing in the human genome? Trends Genet 20: 68-71.

Tress ML, Martelli PL, Frankish A, Reeves GA, Wesselink JJ, Yeats C, Olason PL, Albrecht M, Hegyi H, Giorgetti A, et al. 2007. The implications of alternative splicing in the ENCODE protein complement. Proc Natl Acad Sci 104: 5495-5500.

Uchiyama Y, Takeuchi R, Kodera H, Sakaguchi K. 2009. Distribution and roles of X-family DNA polymerases in eukaryotes. Biochimie 91: $165-170$.
Wang ET, Sandberg R, Luo S, Khrebtukova I, Zhang L, Mayr C, Kingsmore SF, Schroth GP, Burge CB. 2008. Alternative isoform regulation in human tissue transcriptomes. Nature 456: 470-476.

Weis E, Galetzka D, Herlyn H, Schneider E, Haaf T. 2008. Humans and chimpanzees differ in their cellular response to DNA damage and non-coding sequence elements of DNA repair-associated genes. Cytogenet Genome Res 122: 92-102.

Yamtich J, Sweasy JB. 2009. DNA polymerase Family X: Function, structure, and cellular roles. Biochim Biophys Acta 1804: 1136-1150.

Yeo GW, Van Nostrand E, Holste D, Poggio T, Burge CB. 2005. Identification and analysis of alternative splicing events conserved in human and mouse. Proc Natl Acad Sci 102: 2850-2855.

Zhang XH, Chasin LA. 2006. Comparison of multiple vertebrate genomes reveals the birth and evolution of human exons. Proc Natl Acad Sci 103: 13427-13432.

Zhang C, Krainer AR, Zhang MQ. 2007. Evolutionary impact of limited splicing fidelity in mammalian genes. Trends Genet 23: 484-488. 

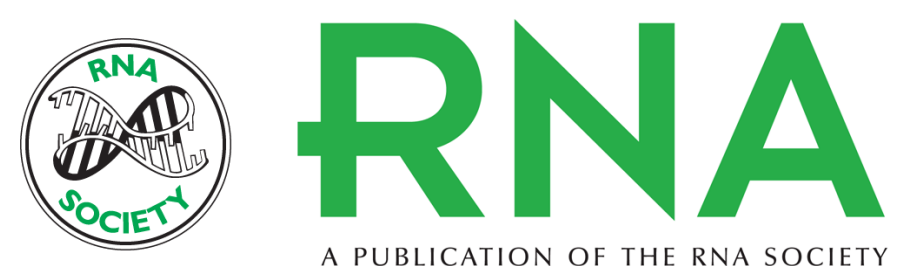

A PUBLICATION OF THE RNA SOCIETY

\section{The adaptive significance of unproductive alternative splicing in primates}

Adonis Skandalis, Mark Frampton, Jon Seger, et al.

RNA 2010 16: 2014-2022 originally published online August 18, 2010

Access the most recent version at doi:10.1261/rna.2127910

\section{References This article cites 35 articles, 7 of which can be accessed free at: http://rnajournal.cshlp.org/content/16/10/2014.full.html\#ref-list-1}

\section{License}
Email Alerting Receive free email alerts when new articles cite this article - sign up in the box at the Service top right corner of the article or click here.

